\title{
CERVICAL SPONDYLOTIC MYELOPATHY
}

\section{Topic Editor: Edward C. Benzel, M.D.}

Cervical spondylotic myelopathy (CSM) is an often underdiagnosed clinical entity. As with most problems requiring surgical intervention, CSM is at times undertreated and at other times overtreated. Cervical spondylotic myelopathy has many manifestations and, therefore, can mimic other disease processes.

The inconsistent use of nomenclature to describe this entity has plagued both the reporting and understanding of CSM. Progress has similarly been stifled by the waxing and waning of symptoms that so often accompany the clinical presentation of CSM. Surgical approaches have, therefore, been chosen in situations in which an optimal result may not be expected and, conversely, they have not been chosen when a good result could have been expected. This confuses the student of CSM and further perverts the literature. The aforementioned waxing and waning clinical course presents significant decision-making dilemmas. This is particularly so in the elderly, in whom the risks of surgery may be significant and the benefits difficult to appreciate.

This issue of Neurosurgical Focus ${ }^{\mathrm{TM}}$ addresses some of these matters. Surgical decision making is discussed in the first manuscript and the consideration of spinal geometry is emphasized. An uncommon entity, the disappearing cervical disc, is addressed in the second manuscript. This condition lends credence to a conservative approach to the decision-making process.

The differential diagnosis of CSM is always relevant; even rare and unlikely "masqueraders" should be considered. The third manuscript addresses the issue of differential diagnosis through a description of CSM associated with a vascular lesion.

Surgical approaches for the management of CSM may seem quite routine. However, multiple variants have been presented in the literature. A technically demanding, unique approach for anterior foraminotomy is described in the fourth manuscript. Finally, a series of patients managed surgically via a ventral corpectomy approach for CSM is presented. The complications and benefits are discussed.

I would like to thank my coreviewers, Paul Cooper, M.D., and Ulrich Batzdorf, M.D., for their diligence and thoroughness with regard to manuscript review. Their expertise, experience, and wisdom contributed substantially to this issue. 